\title{
Traçabilité de l'usage des outils de conception dans un processus collaboratif
}

\author{
Xaviera Calixte $^{1}$, Samia Ben Rajeb $^{2}$, et Pierre Leclercq ${ }^{1}$ \\ ${ }^{1}$ Université de Liège, Place du 20 Août 7, 4000 Liège, Belgique \\ ${ }^{2}$ Université libre de Bruxelles, Avenue Franklin Roosevelt 50, 1050 Bruxelles, Belgique
}

Résumé. Cet article présente un système de traçabilité des usages d'outil (SysTrac) développé par le Lab for User Cognition \& Innovative Design (LUCID), le Collaborative Design and Digital mediations in Architectural Engineering (COLLAeB-BATir) et le Luxembourg Institute of Science and Technology (LIST). Il permet aux concepteurs d'encoder en moins de 5 minutes chacune de leurs activités. Les données récupérées se distinguent en 3 catégories (celles sur l'outil, le temps et l'action). Cet article permet de faire part des premiers constats sur l'opérabilité de cette méthode immersive mise en place pour récupérer la trace des usages des outils des acteurs du projet sur l'ensemble du processus de conception collaborative. La réflexion menée pour créer le SysTrac et son implémentation dans un cas pratique sont détaillées et les premiers résultats y sont illustrés.

Mots-clés. Méthodologie, Conception collaborative, IHM, Récolte des données, Usage des outils.

\begin{abstract}
This article proposes a traceability system tracking the use of tools (SysTrac), developed by the Lab for User Cognition \& Innovative Design (LUCID), the Collaborative Design and Digital mediations in Architectural Engineering (COLLAeB-BATir) and the Luxembourg Institute of Science and Technology (LIST). This system enables the designers to encode each of their activities within minutes. The collected data organize itself in three categories (on the tool, the time and the action). This article presents the first findings on the operability of this immersive method, which is set up to retrieve the track of the tools used by the project stakeholders on the whole collaborative design process. The reflection led to create and implement SysTrac in a practical case is detailed and the first results are illustrated.
\end{abstract}

Keywords. Methodology, Collaborative design, IHM, Data collection, Use of tools. 


\section{Introduction}

L'activité collective au sein du processus de conception n'est pas une thématique récente et différentes théories et études existent sur le sujet. Cependant, le besoin de mélanger les compétences pluridisciplinaires et de travailler à plusieurs autour du même objet n'a jamais été autant d'actualité que depuis ces dernières années (Sklair, 2005).

Différents outils émergent afin d'aider les concepteurs à collaborer et prennent de plus en plus de place dans le mode de fonctionnement des agences, qu'ils apparaissent comme gage de qualité à des étapes du projet ou comme moyen de combler un besoin technique tel que palier les problèmes de la distance physique entre les collaborateurs (Legendre et al., 2016). Se pose alors la question de la pertinence et l'adaptabilité de ces outils aux activités collaboratives en conception.

Cet article propose une méthode immersive de récolte de données centrée sur les usagers dont l'objectif est de pouvoir analyser la place et le rôle des outils utilisés lors d'une conception architecturale collaborative complète. Via cette méthode intégrée dans une interface mobile, l'ensemble des moyens collaboratifs utilisés par les concepteurs a pu être récupéré durant une période de plusieurs mois. Ainsi, les premières parties de cet article présenteront l'état de l'art et la problématique posée. Nous décrirons ensuite le raisonnement qui nous a poussés à développer ce système (partie 4) et la manière dont il a été testé pour la première fois (partie 5). Les dernières parties exposeront nos résultats d'utilisation de la méthode, nos discussions ainsi que les apports, les limites et les perspectives de cette étude.

\section{2. État de l'art}

\subsection{L'outil dans l'activité collective}

Avant de s'interroger sur la place d'un outil dans le processus, il faut s'accorder sur le terme même d' « outil ». Selon le Trésor de la Langue Française informatisé, l' « outil » est défini comme un moyen, au sens large, qui permet d'agir selon un but fixé. L'outil est alors utilisé par l'homme dans le but d'augmenter son efficacité sur une tâche à réaliser. Il permet de simplifier, de rentabiliser, d'optimiser et de mener des actions impossibles sans celui-ci (McLuhan, 1968). Dans le domaine de la conception architecturale, si même un crayon prend dans ce cas le statut d' « outil», il semble donc peu concevable de n'utiliser aucun outil dans le processus. Ce constat semble d'autant plus inévitable lorsque l'on s'intéresse à un processus collectif, la pluralité des acteurs conduisant systématiquement à une multiplication des pratiques.

Plusieurs études catégorisent et structurent les différents outils rencontrés dans un processus de conception. Pour un objectif déterminé et dans un souci d'efficacité propre à une tâche, chaque outil est employé pour différents cas de figure. Certains se complètent et s'enchaînent... C'est en répondant et en qualifiant leur rôle que la place de l'outil est spécifiée au sein d'un processus de conception (Johansen, 1988).

Dans un processus de conception collaboratif, les outils tentent de répondre aux enjeux de la conception et de l'organisation de l'équipe. L'activité collective est articulée autour de 3 grandes actions proposées par Ellis et Wainer (1994) :

- Communiquer : action qui regroupe tous les échanges formels et informels d'information au sujet de l'objet architectural des acteurs de projet ;

- Se coordonner : action qui permet d'organiser le travail collectif;

- Produire : action qui caractérise de manière tangible le projet architectural. 
Ces trois actions s'articulent entre elles tout au long du processus en prenant une place prédominante ou non dans le parcours et les étapes de l'activité collective.

\subsection{Les méthodes pour tracer les usages d'outil dans le processus}

Diverses études reposent sur l'activité collective et différentes méthodes d'observation et d'analyse ont été mises en place. Des principes de codage comme proposé par Gero (1990) ont permis de comprendre le comportement des acteurs, la fonctionnalité de l'objet, des outils sur base de verbatim, du nombre d'échanges observés entre les intervenants et sur un classement de livrables produits. D'autres études portent leur focus sur l'objet et les outils, et cela indépendamment des situations dans lesquelles ils évoluent (Ericsson et Simon, 1993).

Ces deux démarches considérées complémentaires (Dodier, 1995) se focalisent soit sur des objets concrets (production de l'activité), soit sur la fréquence des échanges humains, mais aucune sur l'articulation de ces actions et usages d'outils sur l'ensemble du processus.

L'expérience menée par Otjacques (2008) lui a permis de retracer les échanges d'information en récoltant l'ensemble des e-mails que les acteurs se sont échangés. On retrouve ici une méthode qui a permis de retracer l'usage d'un outil, le logiciel d'envoi des e-mails, sur l'ensemble du processus. Ce travail a permis de comprendre la place et l'usage de l'outil dans les échanges d'information entre concepteurs tout au long du processus.

L'emploi de caméra, la prise de notes libres ou structurées permettent d'observer des situations sans interférer avec l'activité. Ce sont alors des études poussées sur des moments bien précis du processus qui sont analysées (Defays, 2013). Pour permettre de compléter ces informations avec le ressenti du concepteur, des entretiens libres et/ou structurés sont généralement menés.

\section{Problématique}

L'emploi d'outils dans un processus de conception collaborative se base sur l'usage que les concepteurs en font. La posture face à un choix d'outil (pour une tâche à un moment précis) ne peut se comprendre que par le concepteur lui-même parce qu'il mène l'action. De plus, établir un classement des outils sur la base de leurs usages constitue une piste de réflexion originale pour comprendre leur place dans l'activité collective et au sein du processus de conception. Néanmoins, peu de travaux de recherche mettent en lumière les articulations de ces outils entre eux et leurs émergences à l'échelle du processus de conception collaborative dans son intégralité. Alors, l'étude et l'analyse systématique de ces articulations sont nécessaires pour comprendre les choix de chaque concepteur et l'assemblage de tous leurs usages dans le cadre d'un travail collectif durant plusieurs mois.

Nous sommes alors à même de nous poser plusieurs questions. Comment instrumenter le suivi d'un travail de conception collaboratif de manière continue pendant plusieurs mois? Comment récupérer les données sur l'articulation des usages d'outils pour chaque concepteur?

\section{Proposition et méthodologie}

Le besoin de définir et de mettre en place un Système de Traçabilité (SysTrac) en ligne a émergé d'un consortium rassemblant 3 laboratoires qui s'intéressent particulièrement au processus de conception et à l'activité collective, et dont l'objectif est de tracer l'usage de tous les outils employés pendant toute la durée du processus de conception collaborative. 
Ces 3 laboratoires sont : le Lab for User Cognition \& Innovative Design (LUCID), le Collaborative Design and Digital mediations in Architectural Engineering (COLLAeBBATir) et le Luxembourg Institute of Science and Technology (LIST).

Pour répondre aux questions énoncées dans la problématique, la méthode que nous avons mise au point a été construite sur le principe simple du journal de bord, c'est-à-dire le recensement de l'activité dès qu'un outil est utilisé à travers une série de courtes questions. Néanmoins, SysTrac se spécifie par :

- Son mode immersif : lors de la conception, les données sont récupérées dès qu'un outil est utilisé par le concepteur dans le cadre de son processus. Ainsi, chaque outil mis en jeu par un utilisateur participant au projet est étudié de manière individuelle.

- Son emploi régulier, rapide et centré utilisateur : la plateforme joue le rôle de consigne chronologique des usages d'outils. Pour qu'elle puisse être utilisée de manière régulière, celle-ci doit être disponible à n'importe quel moment pour ne pas conditionner son mode d'utilisation et l'encodage ne doit pas prendre plus de 5 minutes.

Pour ce faire, différents scénarii d'usage ont été définis et testés en laboratoire afin de veiller à l'adaptabilité, l'utilisabilité et l'ergonomie de l'interface relativement aux types d'usagers qui auront à l'utiliser. Il était important pour nous de garder aussi leurs motivations afin de garantir un encodage rapide et systématique.

Dans cette même logique, SysTrac ne doit pas interrompre l'activité du concepteur, c'est pourquoi l'encodage se fera une fois l'action terminée.

À chaque utilisation d'outil, quel qu'il soit et quel qu'en soit l'objectif (concevoir, communiquer...), l'utilisateur peut encoder son activité en répondant à une série de sept questions illustrées à la figure 1 et détaillées par la suite selon leurs thématiques.

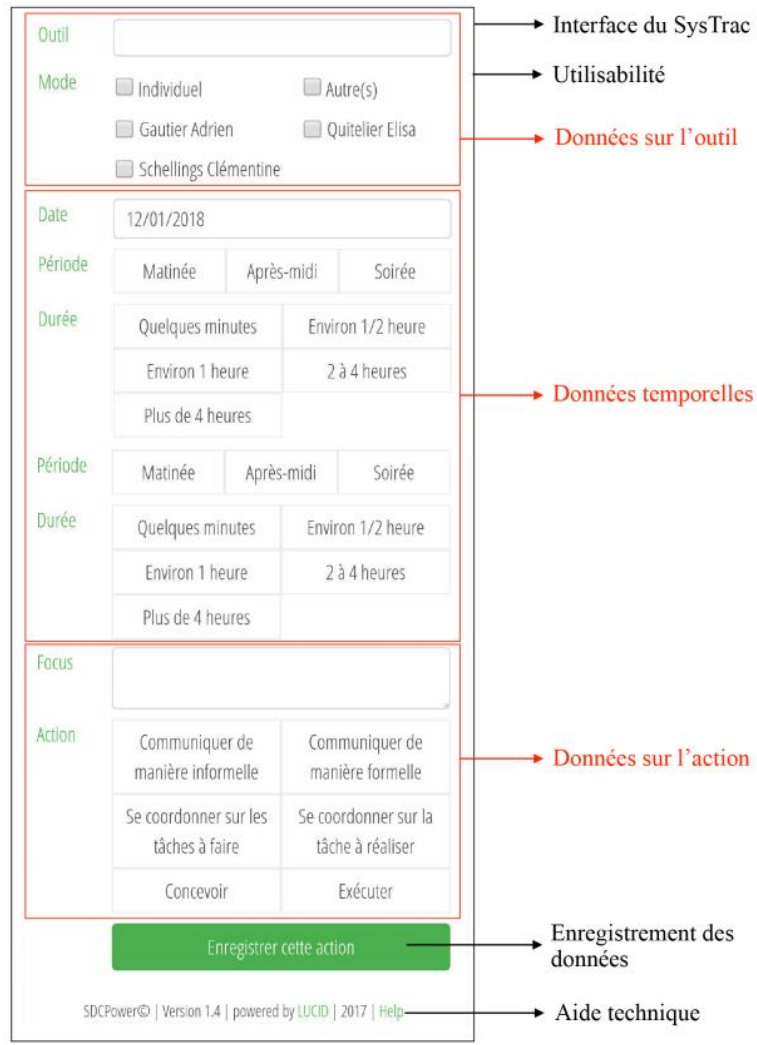

Figure 1. Interface du SysTrac. 


\subsection{Utilisabilité}

Durant la période consacrée à la conception du projet, un profil est créé pour chaque acteur leur permettant d'enregistrer individuellement tous leurs usages d'outils. Ainsi, l'interface est configurée par rapport à la personne identifiée.

Pour proposer un système de récolte de données régulier et rapide, l'utilisabilité du SysTrac a été travaillée sur deux aspects. Le premier point apporté a été de limiter le nombre de questions et de permettre d'y répondre de manière intuitive à l'aide de choix multiples. Le second a été de fournir une interface adaptative sur PC et sur smartphone pour permettre de s'accommoder aux habitudes des usagers.

\subsection{Données sur l'outil}

Le SysTrac devant être complété dès l'usage d'un outil dans le processus de conception du projet, il est compréhensible d'observer une première question se rapportant à l'outil. L'objectif étant de tracer l'usage de chaque outil, le système se focalise sur tous les outils potentiellement utilisés.

La première partie du questionnaire permet donc de récupérer des données factuelles telles que l'« outil » à proprement parler et le « mode» qui permet de savoir avec quel(s) autre(s) membre(s) de l'équipe l'outil a été utilisé pour l'activité, ou si ce dernier a été employé seul.

\subsection{Données temporelles}

Imposer un encodage après chaque emploi d'outil permet de préciser deux notions supplémentaires : la temporalité de l'usage et la succession des outils entre eux.

L'échelle de temps dans cette dynamique est primordiale. Le curseur entre précision et globalité temporelle est un des premiers enjeux de cette méthode de récolte de données. Trois éléments permettent de repositionner l'outil dans l'échelle temporelle du processus :

- La « date » permet d'enregistrer le moment d'utilisation de l'outil.

- La «période » permet à l'utilisateur de repositionner l'usage de l'outil dans la journée en choisissant entre matinée, après-midi ou soirée. Il n'est possible de sélectionner qu'une seule des périodes pour l'encodage, forçant ainsi le concepteur à positionner l'outil dans la période principale d'utilisation. Cette échelle temporelle sur l'ensemble du processus peut paraître trop fine mais, en réalité, elle permet lors de moments d'idées ou de partage d'informations de marquer les changements d'outils sur une même journée.

- La «durée », contrairement aux deux questions précédentes qui permettent de se positionner sur l'échelle de temps du processus, apporte la notion de durée d'utilisation. Certains outils sont utilisés de manière très ponctuelle (action qui ne dure pas dans le temps), ou plus continue. Pour répondre à cette question, il est demandé de faire une sélection parmi les choix suivants : quelques minutes, environ $1 / 2$ heure, environ 1 heure, 2 à 4 heures et plus de 4 heures.

L'information sur la période et celle sur la durée sont complémentaires. La période permet de structurer l'articulation de différents outils utilisés dans la journée. La durée, quant à elle, permet de donner une idée de l'importance temporelle de l'usage. 


\subsection{Données sur l'action}

Pour pouvoir rattacher l'outil sur les activités qui composent le processus, il est nécessaire de nous questionner sur « le pourquoi » de son usage.

Ainsi, en se basant sur base du cadre théorique d'Ellis et Wainer (1994), l'«action» permet de qualifier la tâche à laquelle l'usage de l'outil répond. Néanmoins, une nuance supplémentaire doit être apportée aux trois actions du trèfle. Notre postulat est de penser qu'il existe différents types de communication, de coordination et de production. Ces sousactions, précisées ci-après, se différencient par l'usage de certains outils et par leur moment d'utilisation dans le processus. Le concepteur peut encoder son activité parmi les choix suivants :

- Communiquer :

- communiquer de manière informelle permet d'associer l'outil à des moments d'échange d'information spontanée (exemple : poser une question par e-mail ou par téléphone);

- communiquer de manière formelle englobe toutes les actions qui permettent un échange d'information sur le projet dans un contexte programmé à l'avance (exemple : discuter sur la base d'un ordre du jour lors d'une réunion hebdomadaire).

- Coordonner :

- se coordonner sur les tâches à faire concerne certaines discussions, en dehors des sujets en lien avec le projet à proprement parler et permettant à l'ensemble des acteurs de se coordonner sur les différentes tâches à réaliser ;

- se coordonner sur la tâche à réaliser constitue un complément à la rubrique précédente, où les acteurs se mettent d'accord sur la manière dont une tâche « spécifique » doit être réalisée.

- Produire :

- concevoir regroupe toutes les actions qui concrétisent un artéfact (sous n'importe quelle forme) en pensant à son idéalisation, c'est à dire lorsque le concepteur réfléchit aux aspects fonctionnels, aux solutions de problèmes, à la forme du bâtiment, etc. (exemple : esquisser un croquis d'une idée) ;

- exécuter englobe les actions qui permettent la réalisation d'un artéfact/une production. Plus aucune notion de réflexion sur la conception de l'objet représenté n'est alors en jeu (exemple : mettre au propre le plan du rez-dechaussée).

Pour compléter l'information, une autre question en texte libre, nommée «focus », permet à l'utilisateur d'apporter toute précision pour comprendre l'action qu'il est en train de mener. Ce champ libre d'expression permet d'apporter un complément sur le sujet de la part du concepteur et de limiter le risque de non-considération d'évènements exceptionnels ou non-anticipés qu'engendrent les choix multiples.

\section{Contexte et expérimentation}

Le SysTrac a été utilisé pour la première fois à l'Université de Liège (ULiège), dans un cours de $1^{\text {er }}$ Master de la formation Ingénieur Civil Architecte qui a pour objectif d'apprendre aux étudiants le travail de conception collaborative à distance avec l'École Nationale Supérieure d'Architecture de Nancy. 
Durant 3 mois, 6 groupes de 5 à 6 concepteurs ont participé à la réalisation d'un projet d'architecture. À cette occasion, les acteurs de projet ont été invités à tracer l'usage de leurs outils avec le SysTrac.

Différents moyens ont été mis en place pour motiver les étudiants à encoder régulièrement leurs actions :

- Des rappels hebdomadaires par e-mail ont été envoyés aux étudiants n'ayant encodé aucune activité. Dans le cadre de l'atelier, des revues de projets ont été organisées chaque mercredi entre les étudiants nancéiens et liégeois dans les Studios Digitaux Collaboratifs (Ben Rajeb et Leclercq, 2013). Ainsi, par déduction, chaque semaine, une séance au minimum devait être encodée de la part de tous les étudiants ;

- Une aide informatique est également à disposition pour régler à n'importe quel moment les problèmes dus à la plateforme Web et pour pallier tout problème technique ;

- Les données récoltées par le questionnaire sont également mises à disposition des étudiants de l'ULiège pour les aider dans leurs réflexions sur l'analyse de la collaboration et ainsi leur permettre de réaliser un rapport réflexif sur l'organisation et le déroulement du travail collectif de leur groupe.

- Sur l'ensemble des questionnaires récoltés, un étudiant tiré au sort parmi les participants les plus assidus a pu recevoir une récompense.

À l'issue de la phase des 3 mois de conception collaborative, la récolte des données a été clôturée. Pour appuyer la discussion, des entretiens ont été menés avec certains de ces étudiants. Ceux-ci finissant la phase de conception, leurs retours spontanés nous ont permis de compléter les résultats et les données récupérées par le SysTrac.

\section{Utilisation du SysTrac}

Pour traiter l'ensemble des données récoltées, nous nous servons du logiciel de visualisation Common Tools (Ben Rajeb et Leclercq, 2105) qui permet de générer divers graphes, tels qu'une ligne du temps illustrant le déroulé des actions et l'usage d'outils. Ce logiciel nous permet d'analyser par groupe les différents encodages individuels de chaque acteur. Après l'expérience, nous avons récolté 676 encodages, c'est-à-dire en moyenne 5 heures encodées par acteur et par semaine. Ce premier constat nous pousse à croire que l'emploi du SysTrac ne s'est pas montré trop contraignant dans la pratique. Le tableau 1 reprend les résultats d'encodage après 70 jours.

La date et le nombre d'encodages par acteur étant connus, nous observons aussi que 3 groupes sur 6 ont complété de manière assidue la plateforme durant le processus.

Tableau 1. Le corpus récolté

\begin{tabular}{|l|c|}
\hline \multicolumn{2}{|c|}{ SysTrac - Expérience 1 } \\
\hline \hline Nombre de jours total consacré à l'expérience & 70 jours \\
\hline Nombre total d'encodages & 676 encodages \\
\hline Total d'heures encodées & 1.370 heures \\
\hline Moyenne d'heure / acteur / semaine & $5 \mathrm{~h} /$ acteur / semaine \\
\hline
\end{tabular}

\begin{tabular}{|l|l|l|}
\hline Top 3 des groupes \\
\hline Groupe A - 5 acteurs & 172 encodages & 34 encodages moyen / acteur \\
\hline Groupe B- 6 acteurs & 148 encodages & 25 encodages moyen / acteur \\
\hline
\end{tabular}




\begin{tabular}{|c|c|c|}
\hline Groupe C - 6 acteurs & 110 encodages & 18 encodages moyen / acteur \\
\hline \multicolumn{3}{|l|}{ Top 4 des acteurs } \\
\hline Acteur a & & 69 encodages \\
\hline Acteur b & & 54 encodages \\
\hline Acteur c & & 45 encodages \\
\hline Acteur d & & 40 encodages \\
\hline
\end{tabular}

\section{Discussion}

\subsection{La spontanéité des encodages}

Une fois passée la phase de prise en main de SysTrac par les concepteurs, les résultats sur le nombre d'encodages et les discussions menées avec les étudiants nous renforcent dans l'idée qu'il est nécessaire de travailler en amont le design de l'interface (intuition de réponse, temps de réponse...). Ce constat nous laisse croire que la plateforme est déjà bien calibrée.

Malgré le travail de l'interface et le nombre d'encodages des groupes en fin de processus, l'encodage dans le SysTrac n'est pas pour autant devenu pour la plupart des participants un réflexe après l'usage de chaque outil. Différents rappels ont d'ailleurs été nécessaires tout le long du processus pour inciter les étudiants à compléter la plateforme. La raison évoquée par les étudiants au manque d'encodage est l'oubli.

En instaurant que la prise d'information soit immersive au processus, c'est-à-dire que les données soient transmises par le concepteur dès l'usage d'un outil, les acteurs sont contraints de se concentrer non seulement sur le projet mais aussi sur le travail collaboratif. L'emploi de SysTrac amène chaque individu à se questionner sur les évènements et la nature des actions qu'il est en train de mener. Cette réflexion sur son propre travail n'est pas naturelle. Cette explication amène à penser que ce n'est pas la complexité du codage (d'un point de vue pratique ou de la complexité des thématiques) qui est la cause de la faible fréquence d'emploi du SysTrac.

L'objectif du travail des étudiants n'étant pas focalisé sur le processus de conception, mais bien sur l'objet conçu, il n'est pas évident pour eux de répondre aux questions qui renseignent sur la nature de l'activité. Néanmoins, ce constat renforce la nécessité d'interroger le concepteur sur l'action qu'il est en train de mener à un instant proche de cette action. En effet, si la concentration est grande pour mener une réflexion sur l'action que l'on est en train de mener, il semble dès à présent peu envisageable de récupérer la même précision de données a posteriori du processus.

\subsection{Focus sur l'outil}

Le SysTrac est construit pour tracer les usages des outils, amenant ainsi à détailler la fonction propre liée à chaque emploi d'outil. La réflexion semble pourtant plus instinctive de manière inverse : c'est en s'interrogeant sur le moyen de réaliser une tâche que le choix d'un outil se pose. Pourtant, la démarche développée ici est construite de manière inverse : elle pousse le concepteur a se focaliser d'abord sur l'«outil» employé et à préciser le «pourquoi » de ce choix en imposant une réponse parmi les 6 possibilités d'action (cf. 3.4). Dans le but de récupérer des données autres que le nombre d'outils employés pour une 
action, l'ordre des thématiques abordées sur la plateforme force l'encodeur à se focaliser, une fois l'action menée, sur ce à quoi l'outil a finalement servi pour réaliser la tâche.

De plus, un outil peut être utilisé pour mener différentes tâches lors de son usage. Par exemple : « Je me sers de Skype pour communiquer et me coordonner avec les membres de mon équipe ». Cependant, en imposant aux concepteurs de ne choisir qu'une action par encodage, ils sont forcés de renseigner l'action prioritaire qu'ils sont en train de mener. L'outil peut servir plusieurs besoins et différentes actions par acteur d'un même groupe. On peut observer, lors d'un rendez-vous en visioconférence par Skype avec l'ensemble du groupe, que l'outil, pourtant utilisé par tous au même moment (figure 2), a été utilisé pour des actions différentes par les membres du même groupe (figure 3).

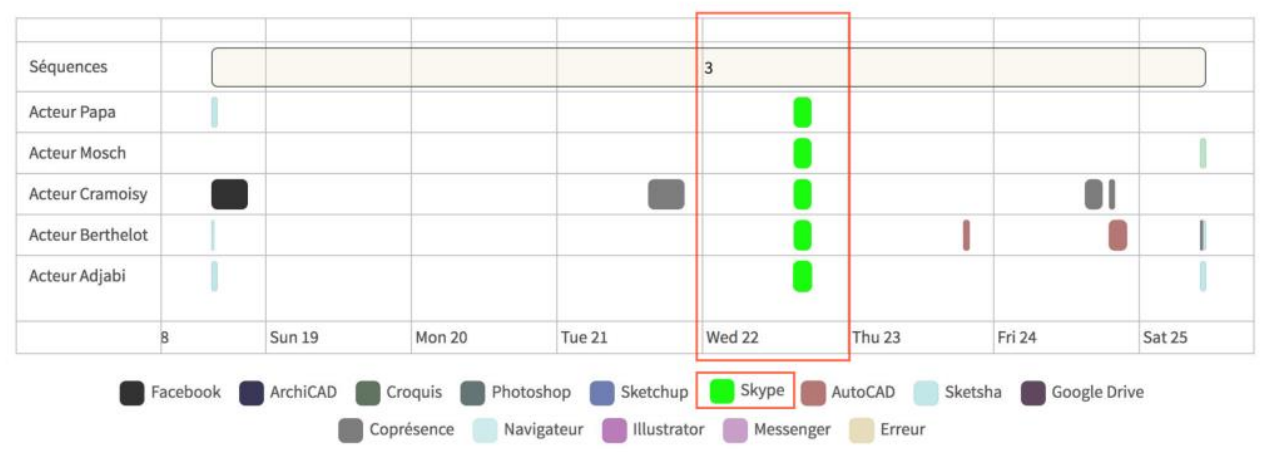

Figure 2. L'usage des outils par tous les acteurs du groupe - ligne du temps.

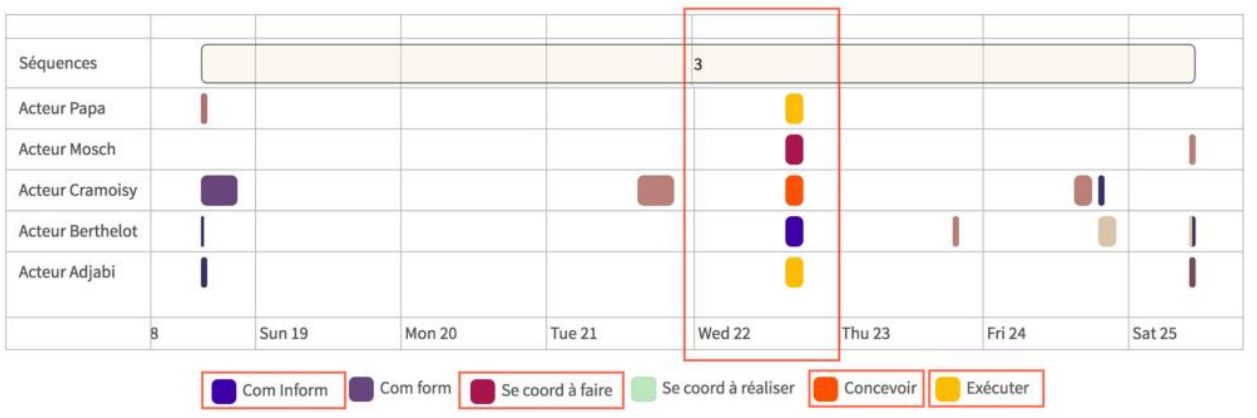

Figure 3. Les actions des acteurs du groupe - ligne du temps.

\subsection{Durée d'utilisation des outils}

Sur base des diagrammes circulaires générés par Common Tools, un deuxième constat ouvre des portes de réflexion pour des analyses prochaines. Le pourcentage en temps de l'usage des différents outils sur l'ensemble du processus et pour tous les groupes confondus, nous permet de constater que les outils comme Skype, Google Drive, Facebook, etc., déclarés comme des outils de communication et de coordination, accumulent $51,51 \%$ du temps encodé tout le long du processus. Néanmoins, lorsque les actions correspondant à la communication et à la coordination sont cumulées, seulement $36,07 \%$ du temps d'encodage y correspond (figure 4).

C'est pourquoi certains outils annoncés comme des instruments à la communication et à la coordination ont été utilisés prioritairement pour un usage non conforme. L'une des 
hypothèses avancées correspond à des détournements d'outils et à des modes de travail spécifiques à des situations propres dans le processus de conception collaborative.
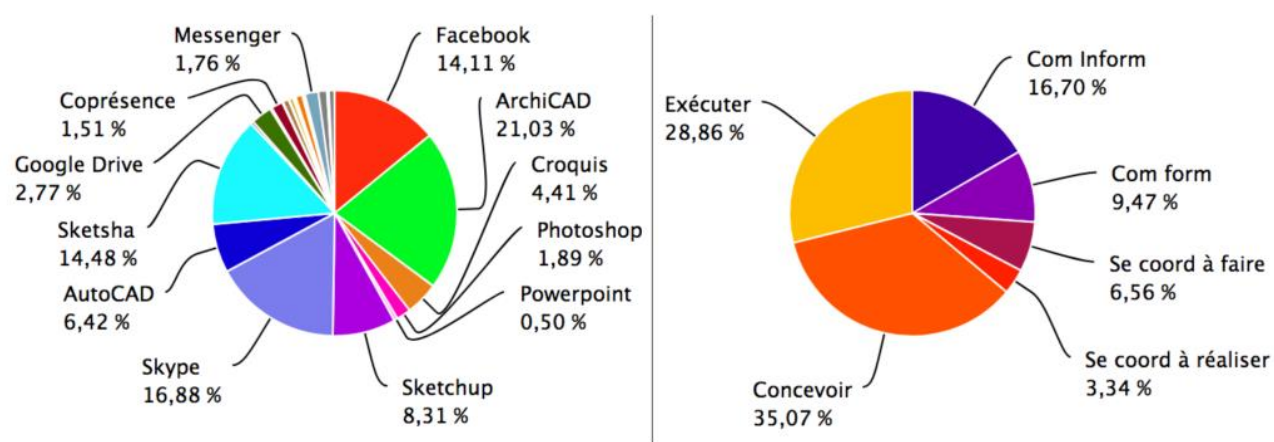

Figure 4. Diagrammes circulaires sur le pourcentage en temps des outils (gauche) et le pourcentage en temps des actions (droite) sur l'ensemble du processus et pour tous les groupes confondus.

Par exemple, parmi les logiciels utilisés, seul le logiciel Sketsha est déclaré au départ comme polyvalent et pouvant répondre aux 3 actions principales spécifiées par Ellis. Sketsha est un logiciel développé au sein du LUCID (Safin, 2012) et permet l'interaction graphique en temps réel et à distance sur bureaux augmentés. Ce logiciel est mis à disposition des étudiants pour les revues hebdomadaires des groupes. Une analyse plus poussée sera menée sur cet outil en particulier pour compléter les recherches et le développement de SysTrac.

\section{Conclusion}

\subsection{Synthèse}

Le système de collecte des données mis en œuvre et présenté dans cet article a permis de récolter une série de données sur l'usage des outils employés tout au long d'un processus de conception collaborative d'une durée de trois mois. Le SysTrac permet à tout acteur de s'identifier par son profil et de compléter la plateforme sur ordinateur ou smartphone. Ainsi, le concepteur, dès la fin d'une tâche, peut encoder en 5 minutes l'activité qu'il vient de mener.

Les données récupérées se structurent en trois grandes thématiques :

- Les données sur l'outil, pour définir quel outil a été utilisé pour la tâche et avec qui ;

- Les données temporelles, pour garder une trace dans le temps de l'usage de l'outil ;

- Et les données sur l'action, pour comprendre la tâche et l'usage de l'outil.

La démarche qui consiste à pousser le concepteur à se questionner sur le «pourquoi » de l'outil, une fois son usage fini, a permis de mettre en évidence qu'un outil peut être employé par l'ensemble du groupe, mais pour des raisons différentes (cela même dans des phases synchrones de conception sur le même outil) et le détournement d'usage de certains d'entre eux.

\subsection{Limites}

Sur l'ensemble du processus au complet, le SysTrac a permis de retracer les usages des outils de l'ensemble des acteurs du projet. La méthode immersive et centrée usager a 
permis de mettre en évidence l'importance de devoir récolter l'information sur le moment même de l'activité. Néanmoins, l'exercice mental qui consiste à analyser ses propres actions au moment où elles se déroulent reste difficile et le réflexe de codage ne s'est pas révélé pour l'ensemble des participants.

\subsection{Perspectives}

Le SysTrac est amené à évoluer pour explorer différentes pistes d'études prochaines sur base des résultats des analyses des données et du retour d'expérience des étudiants.

La démarche est également testée dans un autre contexte que celui pédagogique, le contexte professionnel (toujours dans une démarche de conception collaborative), à travers une équipe de 4 concepteurs lors d'un concours d'architecture. Des réflexions sont menées sur l'insertion de ce type de récolte de données immersif au sein d'agences d'architecture de grande ampleur. Néanmoins, d'autres considérations d'usage et de confidentialité sont à établir pour pouvoir mener à bien l'expérience.

Les auteurs remercient Aurélie Jeunejean, ingénieure informaticienne en charge de la réalisation concrète de la plateforme SysTrac, les 33 étudiants liégeois et nancéiens, ainsi que leurs encadrants pédagogiques pour leur participation à l'expérience menée.

\section{Bibliographie}

Ben Rajeb, S., Leclercq, P. (2013). Apports des configurations spatiales augmentées aux activités de formation par projet. In C. van de Leemput, C. Chauvin, et C. Hellemans (Eds.), Activités humaines, technologies et bien être (pp. 171-178). Paris : Sciences publishing.

Ben Rajeb, S., Leclercq, P. (2015). Instrumented analysis method for collaboration activities. Proceedings of the Fifth International Conference on Advanced Collaborative Networks, Systems and Applications, COLLA 2015, San Julian, Malta.

Defays, A. (2013). Influence des communications multimodales sur le common ground. Proposition d'une méthodologie d'analyse. Liège : http://hdl.handle.net/2268/160251

Dodier, N. (1995). Les Hommes et les Machines : La conscience collective dans les sociétés technicisées, in Collection Leçons de Choses, 1152 (345), Editions Métailié.

Ellis, C., Wainer, J. (1994). A conceptual model of groupware. Chapel Hill, NC.

Ericsson, K.A., Simon H.A. (1993). Protocol Analysis: Verbal Reports as Data, MIT Press, Cambridge.

Johansen, R. (1988). Groupware: Computer support for business teams. New York : The Free Press.

Legendre, A. Lanusse, A., Rauzy, A. (2016). Synchronisation des modèles d'architecture et analyse de risques: quel gain, comment et pourquoi ? $20^{e}$ congrès de maitrise des risques et de sureté de fonctionnement. Saint-Malo.

Gero, J.S. (1990). Design Prototypes : A Knowledge Representation, Schema for Design, in AI Magazine, 11 (4), pp. 26-36.

Mcluhan, C. (1994). Understanding media: the extension of man, $1 \mathrm{sr}$ MIT press ed.

Otjacques, B. (2008). Techniques de visualisation des informations associées à une plateforme de coopération, Namur : https://dial.uclouvain.be/pr/boreal/object/boreal:22707/datastream/PDF_01/view

Safin, S., Juchmes, R., Leclercq, P. (2012). Use of graphical modality in a collaborative design distant setting. In J. Dugdale, C. Masclet, M. A. Grasso, J.-F. Boujut, et P. Hassanaly (Eds.), Proceedings of COOP 2012 : 10th International Conference on the Design of Cooperative Systems. Springer. 
Sklair, L. (2005). The Transnational Capitalist Class and Contemporary Architecture in Globalizing, International Journal of Urban and Regional Research, volume 29,3, 130141. 